\title{
Actividades extraescolares y autoeficacia en niños, niñas y adolescentes: el caso de una escuela en Quito-Ecuador
}

Extracurricular activities and self-efficacy in children and adolescents: the case for a school in Quito-Ecuador

\author{
Diego F. Raza \\ Universidad Andina Simón Bolívar, Ecuador \\ diego.raza@uasb.edu.ec
}

Recibido: 23/01/2019 - Aceptado: 02/11/2019

\section{RESUMEN}

El estudió analizó la diferencia en autoeficacia de estudiantes que se benefician de actividades extraescolares en una escuela pública de la ciudad de Quito. Se comparó la expectativa de autoeficacia de 25 estudiantes que asistieron a actividades extraescolares con la de 85 estudiantes que no tuvieron este estímulo. Para la medición de la autoeficacia se utilizó una versión adaptada para Ecuador del instrumento de Carrasco y Del Barrio. La autoeficacia de los estudiantes que no asistían al centro de actividades extraescolares tuvo una media de 111.47 , frente a una media de 135.88 de los estudiantes que sí asistían al centro de actividades extraescolares. Esta diferencia encontrada resultó significativa estadísticamente con valores de la prueba $\mathrm{t}$ de Student de $\mathrm{t}=$ 4.11 y $p<.001$. Esto significa que, en el caso de la escuela estudiada, los estudiantes que asisten a actividades extraescolares muestran una autoeficacia que es mejor estadísticamente que la de aquellos que no lo hacen y, por tanto, este tipo de actividades debe promoverse entre niños, niñas y adolescentes.

Palabras clave: Actividad extraescolar, autoeficacia, Ecuador 


\begin{abstract}
The study analyzed the difference in self-efficacy of students who benefit from extracurricular activities in a public school in the city of Quito. The expectation of self-efficacy of 25 students who attended extracurricular activities was compared with that of 85 students who did not have this stimulus. For the measurement of self-efficacy, an adapted version for Ecuador of the Carrasco and Del Barrio instrument was used. The self-efficacy of the students who did not attend the center of extracurricular activities had an average of 111.47 , compared to an average of 135.88 of the students who did attend the center of extracurricular activities. This difference was statistically significant with Student's t-test values of $t=4.11$ and $p<0.001$. This means that, in the case of the school studied, students who attend after-school activities show a self-efficacy that is statistically better than those who do not, and therefore this type of activities should be promoted among children and adolescents.
\end{abstract}

Keywords: Extracurricular activities, self-efficacy, Ecuador

\title{
INTRODUCCIÓN
}

Desde muy poco después de la aparición de la obra seminal que introdujo el concepto de autoeficacia (Bandura, 1977), entendida como los juicios que realiza una persona sobre su capacidad de enfrentar una tarea o situación, se realizaron varios estudios e investigaciones que apuntaban a determinar cuáles elementos son los que inciden en su desarrollo. Durante décadas se ha buscado identificar o dar luces sobre la cuestión de cuáles son los determinantes que influyen en el desarrollo de la autoeficacia.

Así, en los años 80 y muy poco después de la publicación de la obra seminal señalada, ya se cuestionaba respecto de los determinantes de autoeficacia en niños y niñas, de manera particular en el contexto académico (Keyser \& Barling, 1981). En este estudio se encontró que la autoeficacia del docente (a diferencia de otros elementos como la estructura de la clase, la participación del estudiante y del docente, las actitudes del profesor, entre otros), tenía incidencia en la autoeficacia de sus estudiantes.

En la década de los 90 continuó esta indagación con una de las publicaciones referentes (Gist \& Mitchell, 1992) que planteó que algunos de los 
determinantes de la autoeficacia tenían que ver con las características de la tarea a enfrentar y su complejidad, junto con el conocimiento y habilidades del sujeto, su condición física y personalidad.

En el nuevo siglo, una de las investigaciones de gran influencia (Schunk \& Pajares, 2002) planteaba que el desarrollo de la autoeficacia en los niños tenía mucho que ver con la influencia familiar que habían recibido, la influencia de otros niños en el aula y, en general, de las prácticas escolares en las que se desenvuelven. En la adolescencia, en cambio, se plantea que los determinantes pueden ser los recursos familiares del joven que tienen relación con su capacidad financiera, ingreso, educación de los padres o círculo social que frecuenta la familia. También, influyen las características de su escuela en cuanto a la estructura de sus procesos de instrucción, nivel de exigencia, retroalimentación que se provee en la escuela, nivel de competencia entre los jóvenes, formas de evaluación, nivel de atención que se recibe por parte de los docentes. Además, la influencia de sus compañeros es especialmente importante en los jóvenes, pues perfila la manera en la que se ve a sí mismo (Schunk \& Meece, 2006).

En la última década han continuado las investigaciones para determinar los factores que inciden en la autoeficacia. Destaca aquella que encontró directamente que la implicación de los padres en la educación de sus hijos (las aspiraciones que tenían respecto de sus logros y su acercamiento proactivo a la escuela), tuvieron incidencia en los niveles de autoeficacia de los jóvenes (Shih, Zheng, Yuan, \& Ford, 2015); así como la investigación que incluso apuntó a factores sociales y culturales como determinantes de la misma (Usher \& Weidner, 2018).

Esta investigación pretende continuar con esta búsqueda, indagando respecto del posible efecto que puede tener la asistencia a actividades extraescolares en la autoeficacia de niños, niñas y adolescentes. Ello en consideración de que estas les dan la posibilidad de regular algunos de los factores que las investigaciones previas han encontrado como determinantes de su autoeficacia: mejor incidencia de un entorno alternativo al familiar con ausencia de adultos en la tarde, interacción mejor controlada con pares, entre otros aspectos. 


\section{ACTIVIDADES EXTRAESCOLARES}

Las actividades extraescolares corresponden a aquellas que se llevan a cabo en un horario distinto al de clase y que tiene como fin -entre otrosbrindar una alternativa de uso del tiempo del estudiante fuera de sus horarios de clase (Lembo \& Ortega, 2005). Con estas actividades se busca otorgar conocimientos y experiencias adicionales, al tiempo que permitir la socialización de los niños, niñas y adolescentes con otros de su edad (Cabo, 2012).

El estudio pionero sobre esta temática, realizado por Coleman (1961), planteaba que la dedicación que el estudiante le preste a sus actividades académicas escolares y a otras actividades fuera de la escuela de carácter social y deportivo, tienen un carácter de suma-cero, en el sentido de que cualquier compromiso con alguno de los tipos de actividad descritos irremediablemente determinaría un compromiso menor con los otros dos tipos de actividades. Es decir, Coleman afirmaba que el compromiso con una actividad extraescolar traía consigo necesariamente una falta o menor compromiso con las actividades académicas escolares y, por lo tanto, un menor desempeño en ellas.

Este planteamiento fue superado con el pasar de las décadas. Actualmente, la postura dominante más bien reconoce el impacto positivo de las actividades extraescolares en los jóvenes, tal como refieren Seow y Pan (2014) en su revisión de la literatura, en la que además incluyen el planteamiento de Broh (2002), quien argumenta que la participación en actividades extraescolares mejora el desempeño escolar por tres vías: ayuda a desarrollar habilidades vitales como la ética de trabajo, perseverancia y otras; mejora el reconocimiento del estudiante por parte de sus pares $y$, por tanto, le facilita la pertenencia a grupos de mejor desempeño académico y, finalmente, contribuye en general a generar lazos sociales con sus compañeros de aula con el consiguiente mejoramiento del capital social del grupo. Asimismo, existe también el planteamiento de otros autores que reconocen el impacto positivo de las actividades extraescolares, hasta cierto punto de dedicación de tiempo, pasado el cual, se podrían generar impactos negativos en el desempeño académico escolar.

Las actividades extraescolares resultan convenientes en familias en las que padre y madre trabajan, sin contar con otro familiar (un hermano 
mayor, una abuela, un tío), que pueda acompañar a los niños y niñas en casa luego de la jornada escolar, ayudarles con sus tareas, vigilar que utilicen el tiempo provechosamente y evitar que se vean expuestos a violencia, consumo de drogas, delincuencia, prostitución, especialmente cuando la familia vive en zonas de tolerancia, marginales y empobrecidas de la ciudad, como lo es el barrio de San Roque (Carvajal \& Romero, 2018).

El importante rol de las actividades extraescolares ha sido ampliamente estudiado en los últimos años; se busca su relación e impacto en las distintas facetas de la vida de niños, niñas y adolescentes alrededor del mundo. Muchos de estos estudios han buscado mostrar su relación con el desempeño de los niños, niñas y adolescentes en la escuela, identificando su relación con el compromiso escolar de los estudiantes (Fredricks, 2011), la mejora de su rendimiento (Rodríguez \& Bakieva, 2011) y de sus resultados escolares en general (Shulruf, 2010; Vukic \& Zrilic, 2016). Se ha indagado, también, sobre el impacto de las actividades extraescolares en mejorar la pertenencia grupal como factor de protección en la adolescencia (Ruvalcaba, Gallegos, Borges, \& Gonzalez, 2017), así como en fortalecer las relaciones intergrupales e interraciales en la escuela secundaria (Knifsend \& Juvonen, 2017), en promover acciones prosociales en los jóvenes (Villarreal \& Gonzalez, 2016), así como en prevenir el acoso escolar (Riese, Gjelsvik, \& Ranney, 2015) y prevenir problemas de conducta disruptiva en adolescentes (Driessens, 2015). Las actividades extraescolares de niños, niñas y adolescentes, se han relacionado incluso con la construcción de su propia auto-determinación (Vinoski, Graybill, \& Roach, 2016), mejoramiento en su autoestima (Kort-Butler \& Hagewen, 2011), en su desarrollo moral cognitivo (Brown-Liburd \& Porco, 2011) e, incluso, en general con su salud mental (Lushington et al., 2015). Esta investigación busca identificar si la participación de niños, niñas y adolescentes en activides extraescolares determina diferentes niveles en su autoeficacia.

\section{AUTOEFICACIA}

El mejor concepto de autoeficacia es aquel que ofrece Bandura (1987), cuando la define como "los juicios que cada individuo hace sobre sus capacidades, en base a los cuales organizará y ejecutará sus actos de modo que le permitan alcanzar el rendimiento deseado" (p. 373). Estos juicios que hace el individuo sobre sus capacidades inciden en el denuedo y optimismo con el que se aplicará para el logro de sus objetivos y superación 
de dificultades, en la regulación de su bienestar psicológico y en su capacidad de responder a altos niveles de estrés (Barraza, 2010). La autoeficacia de una persona puede ser general cuando hay el convencimiento de poder enfrentar los desafíos de la vida en los términos más amplios, o específica, cuando se refiere a una situación en particular (Luszczynska, Scholz, \& Schwarzer, 2005).

En este estudio, el análisis se limitará a la autoeficacia de niños, niñas y adolescentes, según lo plantean Carrasco y Del Barrio (2002); con sus componentes relativos a lo académico, como creencia de poder superar los retos de la vida académica (Putwain, Sander, \& Larkin, 2013); a lo social, entendido como creencia de poder construir y mantener relaciones sociales mutuamente beneficiosas y superar conflictos con otras personas (Bandura, Caprara, Barbaranelli, Pastorelli, \& Regalia, 2001); y a lo autorregulatorio, como seguridad de poder resistir la presión social y no cometer conductas inadecuadas (Pastorelli et al., 2001).

El presente estudio se propuso verificar la existencia de niveles de autoeficacia mayores en los sujetos que asisten regularmente a actividades extraescolares en una escuela de Quito.

\section{MÉTODO}

\section{Participantes}

Se evaluaron a todos los niños de una escuela pública en Quito cuyas edades se encontraban entre los 8 y 15 años y que cursaban desde el cuarto nivel hasta el décimo nivel de Educación General Básica (EGB) en el año lectivo 2014 - 2015. El número de niños, niñas y adolescentes evaluados ascendió a 110, de los cuales 25 asistían al centro de actividades extraescolares que se consideró para este estudio. Los otros 85 niños no asistían a ninguna actividad extraescolar.

De los 110 niños, niñas y adolescentes, 59 fueron de sexo masculino y 51 de sexo femenino. Su edad tuvo un promedio de 9.47 años, con una DT = 1.13. Todos los sujetos de la muestra viven en el mismo barrio de la ciudad aledaño a la escuela en donde se tomó la muestra. Se trata de un sector empobrecido, lleno de ventas ambulantes, presencia de indigentes, con expendio clandestino de licor artesanal y drogas, acumulación de basura, 
robos y prostitución (Carvajal \& Romero, 2018). Los sujetos de la muestra, además, no cuentan en su hogar con una persona adulta que les pueda apoyar directamente en sus tareas escolares luego de la jornada escolar.

\section{Instrumento}

Para evaluar la expectativa de autoeficacia de los dos grupos (los 25 niños, niñas y adolescentes que asistían al centro de actividades extraescolares y los 85 niños, niñas y adolescentes que no lo hacían), se utilizó una versión adaptada a Ecuador del test Evaluación de la autoeficacia en niños y adolescentes (Carrasco \& Del Barrio, 2002).

La adaptación del test determinó ajustar el léxico para hacerlo más comprensible para los niños, niñas y adolescentes ecuatorianos que debían responder el test, así como fue necesario cambiar el nombre de ciertas asignaturas que se las conoce en Ecuador de una manera distinta que en España.

El test adaptado se puede consultar en Raza (2017) e incluye tres dimensiones (autoeficacia académica, social y autorregulatoria), que se miden a través de 35 preguntas que indagan sobre cómo percibe el sujeto su propia capacidad de desempeñar varias actividades académicas (hacer deberes, concentrarse, tomar apuntes, organizar su trabajo, tener éxito en sus distintas asignaturas); sociales (hacer amigos, participar en equipos deportivos, conversar con sus pares, trabajar en grupo, entre otras); y de autorregulación (frente a la presión por fumar, hacer cosas incorrectas o prohibidas en la escuela).

Cada pregunta se contesta con una escala tipo Likert con cinco opciones: 1 (fatal), 2 (no tan bien), 3 (bien), 4 (muy bien) y 5 (excelente). La validez del test se midió a través de la prueba $V$ de Aiken en la que se obtuvieron valores mayores a .80 en cada pregunta. Su fiabilidad fue medida a través del alfa de Cronbach que fue de .74 .

\section{Procedimiento}

Se buscó una entrevista con los directivos de la escuela, a través de los cuales se gestionó la autorización para que los niños participen llenando el instrumento de evaluación en sus propias aulas de clase. Los mismos directivos informaron cuáles de esos niños, niñas y adolescentes asistían 
al centro de actividades extraescolares, lo que fue confirmado posteriormente con el mismo centro. Los directivos del centro informaron además que se contaba con autorización de los padres de familia para aplicar este tipo de preguntas a los estudiantes.

\section{Análisis estadístico}

Para la comparación de la autoeficacia en los dos grupos, se inició aplicando pruebas de normalidad de Shapiro Wilk para determinar la necesidad de aplicación de pruebas paramétricas o no paramétricas. Luego se aplicó la prueba t de Student para probar la significación estadística a las diferencias encontradas en la media de la variable estudiada en los dos grupos.

\section{RESULTADOS}

La distribucion de la variable autoeficacia en el grupo de estudiantes que no asisten al centro de actividades extraescolares fue normal (Shapiro WiIks $Z=-.109, p=.543$ para el grupo de 85 sujetos), al igual que la distribución de la variable en el otro grupo (Shapiro Wilks $Z=.744, p=.228$ ). Para confirmar que es pertinente la comparación de medias, se aplicó además una prueba de Levene a los datos utilizados. Se encontró un valor $F=1.68$ $\mathrm{yt}=.1502$, que confirman que las varianzas de los dos grupos son iguales y que por tanto es aplicable la prueba paramétrica de $t$ de Student para comparación de medias.

Las diferencias en la autoeficacia (total) y sus componentes de autoeficacia académica, social y autorregulatoria, entre niños, niñas y adolescentes que asisten al centro y aquellos que no lo hacen, se resume en la Tabla 1.

Tabla 1

Autoeficacia y sus componentes, media y desviación estándar

\begin{tabular}{lcccc}
\hline & \multicolumn{2}{c}{$\begin{array}{c}\text { Grupo que asiste al } \\
\text { centro }\end{array}$} & \multicolumn{2}{c}{$\begin{array}{c}\text { Grupo que no asiste } \\
\text { al centro }\end{array}$} \\
& $\boldsymbol{M}$ & $\boldsymbol{D T}$ & $\boldsymbol{M}$ & $\boldsymbol{D T}$ \\
\hline Autoeficacia Académica & 67.76 & 1.77 & 54.83 & 1.42 \\
Autoeficacia Social & 50.52 & 1.78 & 43.05 & 1.26 \\
Autoeficacia Autorregulatoria & 17.60 & 1.08 & 13.59 & 0.70 \\
Autoeficacia (total) & 135.88 & 4.22 & 111.47 & 2.97 \\
\hline
\end{tabular}


La autoeficacia de los niños, niñas y adolescentes que no asisten al centro de actividades extraescolares tuvo $M=111.47$, frente a $M=135.88$ de los niños, niñas y adolescentes que sí asisten al centro de actividades extraescolares.

La diferencia encontrada en la autoeficacia (111.47 versus 135.88), resultó significativa estadísticamente con valores de la prueba t de Student a una cola de $t=4.11$ y $p<.001$, con un intervalo de confianza del $95 \%$. El tamaño de esta diferencia además es considerable con un valor de Cohen - $d$ $=.93$, y un poder estadístico igual a 1.0, lo que abona en la seguridad de interpretación de los resultados. Esto significa que, en el caso de la escuela estudiada, la asistencia de los estudiantes a actividades extraescolares, mejora su autoeficacia.

\section{DISCUSIÓN Y CONCLUSIONES}

El resultado final de este estudio es que los niños, niñas y adolescentes que realizan actividades extraescolares, en comparación de aquellos que no realizan actividades extraescolares, muestran niveles más altos de autoeficacia en general, compuesta por dimensiones de autoeficacia social, autorregulatoria y académica.

Este resultado complementa lo planteado en los estudios previos de Canchigña (2011), Cevallos y Pilco (2012), Rodríguez y Bakieva (2011), Kort-Butler y Hagewen (2011), que muestran efectos positivos de las actividades extraescolares en los estudiantes que participan en ellas, pues mejoran su percepción sobre el propio nivel de aprendizaje, su inteligencia lógica y matemática, su rendimiento académico e incluso su autoestima.

Con este estudio se puede, entonces, abonar con más elementos que justificarían la asistencia de estudiantes a actividades extraescolares, pues además de los resultados mencionados de estudios previos, esta investigación sugiere que también la autoeficacia podría mejorarse en los niños, niñas y adolescentes que atienden a estas actividades. Por esta razón y con base en los resultados obtenidos en la escuela analiza$\mathrm{da}$, parece conveniente ampliar futuras investigaciones para identificar lo que sucede en otras escuelas de la ciudad y el país, en relación con posible mejora de la autoeficacia por asistencia a actividades extraescolares de niños, niñas y adolescentes. 
El acompañamiento extraescolar que recibieron los sujetos de este estudio consiste en un conjunto de actividades de tutela, guía en deberes escolares, lectura compartida de textos selectos, ejercicios matemáticos y de escritura, provisión de espacios para reposo y descanso durante la tarde, así como práctica deportiva. Tal vez también deba indagarse a futuro si existe diferencia en el efecto de estas distintas actividades en los sujetos de estudio y distinguir esos efectos por su edad y género.

Para finalizar, se debe señalar que si bien la información que aporta esta investigación es valiosa, no deben perderse de vista las limitaciones de este estudio, referidas de una parte a que abarcó nada más que una escuela en la ciudad de Quito, que si bien puede considerarse similar al resto de escuelas públicas del país (pues no tiene ninguna particularidad que la haga especial o diferente), es tan solo un caso de estudio.

\section{REFERENCIAS}

Bandura, A. (1977). Self-efficacy: Toward a unifying theory of behavioral change. Psychological Review, 84(2), 191-215. doi: 10.1037/0033-295X.84.2.191

Bandura, A. (1987). Pensamiento y acción: Fundamentos sociales. Martínez Roca.

Bandura, A., Caprara, G. V., Barbaranelli, C., Pastorelli, C., \& Regalia, C. (2001). Sociocognitive self-regulatory mechanisms governing transgressive behavior. Journal of Personality and Social Psychology, 80(1), 125-135. doi: 10.1037/0022-3514.80.1.125

Barraza, A. (2010). Validación del inventario de expectativas de autoeficacia académica en tres muestras secuenciales e independientes. Revista CPU-e, (10), 1-30.

Broh, B. A. (2002). Linking Extracurricular Programming to Academic Achievement: Who Benefits and Why? Sociology of Education, 75(1), 6995. doi: $10.2307 / 3090254$

Brown-Liburd, H. L., \& Porco, B. M. (2011). It's What's Outside that Counts: Do Extracurricular Experiences Affect the Cognitive Moral 
Development of Undergraduate Accounting Students? Issues in Accounting Education, 26(2), 439-454. doi: 10.2308/iace-10022

Cabo, C. (2012). El "homeschooling" en España: Descripción y análisis del fenómeno. Recuperado de http://www.tdx.cat/handle/10803/94200

Canchigña, F. (2011). Los trabajos extracurriculares en el aula influyen en el aprendizaje de los séptimos años de educación básica paralelos $A$ y B de la Escuela Fiscal Mixta "CASPICARA", de la parroquia Sangolquí, Cantón Rumiñahui, Provincia de Pichincha (Tesis inédita de Pregrado). Universidad Técnica de Ambato, Ecuador. Recuperado de http://repo.uta.edu.ec:8080/xmlui/handle/123456789/3518

Carrasco, M. Á., \& del Barrio, M. V. (2002). Evaluación de la autoeficacia en niños y adolescentes. Psicothema, 14(2), 323-332.

Carvajal, A. M., \& Romero, D. (2018, diciembre 24). El barrio quiteño San Roque afronta cinco problemas a diario. El Comercio. Recuperado de https://www.elcomercio.com/actualidad/barrio-san-roque-problemas-seguridad.html

Cevallos Veintimilla, M. R., \& Pilco Guailla, F. D. (2012). Las tareas extracurriculares para desarrollar la inteligencia lógico matemática con los niños y niñas del séptimo año de Educación General Básica de la Escuela "Fray Vicente Solano" de la parroquia Central del cantón Chillanes, provincia de Bolívar, en el periodo lectivo 2011 - 2012. (Tesis inédita de Licenciatura). Universidad estatal de Bolívar, Ecuador. Recuperado de http://www.biblioteca.ueb.edu.ec/ handle/15001/1183?mode=full

Coleman, J. S. (1961). The adolescent society: The social life of the teenager and its impact on education. New York: Free Press of Glencoe.

Driessens, C. M. E. F. (2015). Extracurricular activity participation moderates impact of family and school factors on adolescents' disruptive behavioural problems. BMC Public Health, 15(1), 1-13. doi: 10.1186/ s12889-015-2464-0 
Fredricks, J. A. (2011). Engagement in School and Out-of-School Contexts: A Multidimensional View of Engagement. Theory Into Practice, 50(4), 327-335. doi: 10.1080/00405841.2011.607401

Gist, M. E., \& Mitchell, T. R. (1992). Self-Efficacy: A Theoretical Analysis of Its Determinants and Malleability. The Academy of Management Review, 17(2), 183. doi: 10.2307/258770

Keyser, V., \& Barling, J. (1981). Determinants of children's self-efficacy beliefs in an academic environment. Cognitive Therapy and Research, 5(1), 29-39. doi: 10.1007/BF01172324

Knifsend, C. A., \& Juvonen, J. (2017). Extracurricular Activities in Multiethnic Middle Schools: Ideal Context for Positive Intergroup Attitudes? Journal of Research on Adolescence (Wiley-Blackwell), 27(2), 407422. doi: 10.1111/jora.12278

Kort-Butler, L., \& Hagewen, K. (2011). School-Based Extracurricular Activity Involvement and Adolescent Self-Esteem: A Growth-Curve Analysis. Journal of Youth \& Adolescence, 40(5), 568-581. doi: 10.1007/ s10964-010-9551-4

Lembo, C., \& Ortega, N. (2005). Las actividades extraescolares y su vinculación con la formación académica. México: Universidad Pedagógica Nacional.

Lushington, K., Wilson, A., Biggs, S., Dollman, J., Martin, J., \& Kennedy, D. (2015). Culture, Extracurricular Activity, Sleep Habits, and Mental Health: A Comparison of Senior High School Asian-Australian and Caucasian-Australian Adolescents. International Journal of Mental Health, 44(1/2), 139-157. doi: 10.1080/00207411.2015.1009788

Luszczynska, A., Scholz, U., \& Schwarzer, R. (2005). The General Self-Efficacy Scale: Multicultural Validation Studies. Journal of Psychology, 139(5), 439-457.

Pastorelli, C., Caprara, G. V., Barbaranelli, C., Rola, J., Rozsa, S., \& Bandura, A. (2001). The structure of children's perceived self-efficacy: A cross-national study. European Journal of Psychological Assessment, 17(2), 87-97. doi: 10.1027//1015-5759.17.2.87 
Putwain, D., Sander, P., \& Larkin, D. (2013). Academic self-efficacy in study-related skills and behaviours: Relations with learning-related emotions and academic success. British Journal of Educational Psychology, 83(4), 633-650. doi: 10.1111/j.2044-8279.2012.02084.x

Raza, D. F. (2017). Formación extracurricular en valores en la expectativa de autoeficacia de estudiantes del cuarto al décimo nivel de educación general básica de una escuela fiscal en Quito Ecuador, 20142015. Universidad de Posgrado Norbert Wiener, Perú.

Riese, A., Gjelsvik, A., \& Ranney, M. L. (2015). Extracurricular Activities and Bullying Perpetration: Results From a Nationally Representative Sample. Journal of School Health, 85(8), 544-551. doi: 10.1111/ josh. 12282

Rodríguez, C. C., \& Bakieva, M. (2011). Actividades extraescolares y rendimiento académico: Diferencias en autoconcepto y género. Revista de Investigación Educativa, 29(2), 447-465.

Ruvalcaba, N. A., Gallegos, J., Borges, A., \& Gonzalez, N. (2017). Extracurricular activities and group belonging as a protective factor in adolescence. Actividades extraescolares y pertenencia al grupo como factor protector en la adolescencia, 23(1), 45-51. doi: 10.1016/j. pse.2016.09.001

Schunk, D. H., \& Meece, J. (2006). Chapter 3: Self-efficacy development in adolescence. En T. Urdan \& F. Pajares, SelfEfficacy Beliefs of Adolescents. IAP.

Schunk, D. H., \& Pajares, F. (2002). Chapter 1-The Development of Academic Self-Efficacy. In A. Wigfield \& J. S. Eccles (Eds.), Development of Achievement Motivation (pp. 15-31). doi: 10.1016/ B978-012750053-9/50003-6

Seow, P. S., \& Pan, G. (2014). A Literature Review of the Impact of Extracurricular Activities Participation on Students' Academic Performance. Journal of Education for Business, 89(7), 361-366. doi: 10.1080/08832323.2014.912195 
Shih, H. R., Zheng, W., Yuan, P. C., \& Ford, R. T. (2015). Development of Self Regulation Skills and Academic Self-Efficacy Beliefs. V005T05A008V005T05A008. doi: 10.1115/IMECE2015-50343

Shulruf, B. (2010). Do extra-curricular activities in schools improve educational outcomes? A critical review and meta-analysis of the literature. International Review of Education / Internationale Zeitschrift für Erziehungswissenschaft, 56(5/6), 591-612. doi: 10.1007/ s11159-010-9180-x

Usher, E., \& Weidner, B. (2018). Chapter 7: Sociocultural influences on self-efficacy development. In G. A. D. Liem \& D. M. Mclnerney (Eds.), Big Theories Revisited 2. IAP.

Villarreal, V., \& Gonzalez, J. E. (2016). Extracurricular Activity Participation of Hispanic Students: Implications for Social Capital Outcomes. International Journal of School \& Educational Psychology, 4(3), 201-212.

Vinoski, E., Graybill, E., \& Roach, A. (2016). Building Self-Determination through Inclusive Extracurricular Programs. TEACHING Exceptional Children, 48(5), 258-265.

Vukic, V. V., \& Zrilic, S. (2016). The Connection between Pupils' School Success and Their Inclusiveness in Extracurricular and Out-of-School Activities in Croatia. World Journal of Education, 6(3), 29-38. 